\section{Vol. 66, No. 1}

In the report "Guidance for Assessment of Poliovirus Vaccination Status and Vaccination of Children Who Have Received Poliovirus Vaccine Outside the United States," on page 24, under the section "Children with documentation of poliovirus vaccination." the first paragraph should have read as follows:

Previous poliovirus vaccination is valid if documentation indicates receipt of IPV or tOPV. tOPV was used for routine poliovirus vaccination before April 1, 2016 in all OPV-using countries. Therefore, if a child has documentation of receipt of an OPV dose (rather than "tOPV”) before April 1, 2016, this represents a tOPV dose and should be counted towards the U.S. vaccination schedule, unless specifically notated that it was administered during a vaccination campaign.* Consistent with the polio eradication strategy, doses of OPV administered on or after April 1, 2016 are either bOPV (used in routine vaccination and campaigns), or mOPV (used in a type-specific outbreak response); these doses do not count towards the U.S. vaccination requirements for protection against all three poliovirus types. Persons aged $<18$ years with doses of OPV that do not count towards the U.S. vaccination requirements should receive IPV to complete the schedule according to the U.S. IPV schedule.

${ }^{*} \mathrm{mOPV}$ or bOPV were often used in vaccination campaigns but doses administered during vaccination campaigns are not typically recorded in parent-held records. These doses do not count towards the U.S. vaccination requirements. 